\title{
USING A SOFT SYSTEMS METHODOLOGY FRAMEWORK TO GUIDE THE CONCEPTUAL MODELING PROCESS IN DISCRETE EVENT SIMULATION
}

\author{
José Arnaldo Barra Montevechi \\ Jonathan Daniel Friend \\ Federal University of Itajubá \\ Avenida BPS, 1303, Bairro Pinheirinho \\ Itajubá, Minas Gerais, 37500 903, BRAZIL
}

\begin{abstract}
Conceptual modeling (CM) for simulation has traditionally been described as a highly qualitative process which is difficult to define. Previous articles have investigated the use of Soft Systems Methodology (SSM), a problem structuring method, in healthcare simulation studies in order to define general system understanding, study objectives, and model content. However, no article has proposed the use of SSM in order to guide all CM phases. This article presents a theoretical discussion involving SSM in simulation and discussion on the methodology's knowledge acquisition capabilities and contributes to simulation literature by proposing a step-by-step SSM-CM approach, based on a combination of previously established SSM-CM techniques from scientific literature, in order to guide all conceptual modeling phases. A SSM view to simulation project management is provided, which may help simulation practitioners to structure project activities while also identifying good CM practices.
\end{abstract}

\section{INTRODUCTION}

Conceptual modeling (CM) in simulation may be seen as a compass, initially orienting simulation practitioners and their collaborators as they establish the direction of their efforts throughout the steps of process mapping, data collection and analysis, computational modeling and eventual optimization. A wellguided CM process will define the study's context, research objectives, and model components and assumptions, thus aiding researchers to arrive at their goals; however, if the CM process gets off track, it is likely that researchers will lose their bearing and results of the entire simulation project will end up off the mark.

One seemingly inherent challenge to $\mathrm{CM}$ is that it is often a highly qualitative process involving interviews, discussion, meetings and observations (rather than spreadsheets, statistics and mathematical modeling), thus skirting the typically quantitative scope of other steps involved in operational research and simulation projects.

Robinson (2008a) asserts that CM is the most important phase for achieving specific simulation research goals. Law (1991) was one of the first to state that CM is more of an art than a science. Law (2009) later defined the step of choosing the level of model detail, which depends on project objectives, system performance measures and data availability, as the artistic aspect of CM. Due to the typically ill-defined nature of the CM process, simulation practitioners - especially newcomers to the area - can get lost in the details, such as which aspects to include, to exclude, what to measure and where to direct their attention and efforts.

Soft Systems Methodology (SSM) (Checkland 1999) offers a set of tools with which to overcome this hurdle for CM in simulation. The creator of SSM, Checkland (1999), alleges that the methodology helps 


\section{Montevechi and Friend}

define messy problems in complex systems which rely heavily on human interaction. He also emphasizes that, within SSM terminology, a system does not refer to a logical or physical structure, but rather to the process of investigation in itself. Described by its creator as a structured learning system (Checkland 1999) and by Kotiadis (2007) as a problem structuring method (PSM), SSM aims to transparently structure the understanding process undertaken by researchers. It is worth mentioning here that SSM was initially molded by Checkland in the 1970s as a means by which to approach operational research projects in the industrial sector (Checkland and Scholes 1999).

Although not directly developed for simulation projects, SSM has been adapted by multiple authors in different steps of the CM process in simulation (Lehaney and Paul 1994, Lehaney, Paul, and Clarke 1999, Kotiadis 2007, to name a few) due to its ability to structure the highly qualitative inquiry process and acquire individual-specific knowledge while also assimilating different viewpoints on the same investigation system.

Although the previously stated authors successfully established the link between SSM and simulation project management in separate CM phases, no articles have been found by the authors of this paper which propose an SSM application for the entire knowledge acquisition process. Thus, the objective of this paper is to present two previous SSM approaches to CM in simulation, and discuss the potential for molding these two methods into a streamlined approach to be used, from the initial step of problem definition all the way to defining model content and assumptions, while also focusing on how SSM can contribute to overall simulation project management by helping define the initial direction of the simulation project from its outset.

Following this introduction, this paper is structured in the following sequence. The second section presents SSM and the CM process and shows relevant literature. The third section gives a quick runthrough of conventional SSM tools in order to clarify the context, specific terminology and subsequent references in the text. The fourth section presents previous SSM applications in CM for simulation in further detail. The fifth section proposes a five-step SSM framework which aims to encompass the CM process, followed by a sub-section detailing an application of the framework in an action-research study. The sixth section presents discussion on how SSM and CM relate to overall simulation project management, and the final section presents the authors' conclusions and recommendations for future research in the area of SSM and simulation projects.

\section{SOFT SYSTEMS METHODOLOGY AND CONCEPTUAL MODELING}

A conceptual model, used for the purpose of simulation projects, can be defined as a mathemati$\mathrm{cal} /$ logical/verbal, non-computational representation of the problem entity for a simulation study (Sargent 2011). It is an abstraction of the real-world system which is to be analyzed. Balci, Arthur and Nance (2008) have asserted that, due to the ever-expanding computational capacity of simulation software packages, and therefore their ability to handle complex analyses, CM serves the purpose of organizing, synthesizing and conserving complex system knowledge and understanding for continuing simulation projects.

That CM serves as a guide in simulation project elaboration can be affirmed by Wang and Brooks (2007), who showed that experienced practitioners spend more time discussing system behavior and documenting conceptual models. In their study, which looked at groups of experienced and novice simulation practitioners, the experienced practitioners had an easier time statistically validating their computational models, suggesting a link between conceptual modeling and the later steps of computer model validation.

In spite of its apparent importance, simulation textbooks generally skim over CM and jump straight into programming and software-specific questions (Chwif and Medina 2007). However, this is not to say that CM has not been examined over the years. Recently, Robinson (2008b) proposed a documentation method for CM-specific knowledge; Law (2009), an assumptions document. As a few examples, Montevechi et al. (2010), Karagöz (2008), Chwif and Medina (2007) and Adbdulmalek and Rajgopal (2007) have either put forth or purport different CM modeling techniques. 


\section{Montevechi and Friend}

All of these studies mentioned have suggested different, valid means by which to organize and represent the knowledge acquired during simulation projects; however, very few studies have addressed the actual question of how to acquire knowledge.

Kotiadis and Robinson (2008) affirm that two important phases of CM are: Knowledge Acquisition and Model Abstraction. Only through sound knowledge acquisition can a validated conceptual model be created. The cited authors state that SSM is directly related to knowledge acquisition, but that it also plays a role in model abstraction (which would be conceptual model construction using a CM technique).

According to Robinson (2008b), the CM process consists of a series of five phases:

I. Developing an understanding of the problem situation,

II. Determining modeling objectives,

III. Identifying model outputs (responses),

IV. Identifying model inputs (experimental factors),

V. Determining model content (scope and level of detail), assumptions and simplifications.

Robinson (2008a) defines model outputs as the responses of a simulation; or rather, the output is the response variable in question (i.e. throughput, number of clients served, number of patients treated). Model inputs consist of elements that may be altered to improve or better understand a problem. In other words, inputs are experimental factors. Model content refers to the model's components visually and logically represented in the model, and their interconnections, aside from assumptions and simplifications made. All of these components represent system intricacies which researchers must be able to understand. Formalizing knowledge acquisition could facilitate this process.

Soft Systems Methodology, a problem structuring method, offers a structured series of phases, which is meant to guide the qualitative inquiry process involved in complex problem definition. Among the tools proposed by Checkland (1999) are: Rich Pictures, 123 analyses, root definitions, the mnemonic CATWOE and Conceptual Models (which, in order to avoid confusion, will be referred to as Purposeful Activity Models or PAM, as proposed by Kotiadis (2007), in the rest of the paper). Relevant SSM tools will be discussed herein; however, for further reading, interested readers should consult Checkland (1999) and Checkland and Scholes (1999).

In the literature, academic production on SSM in simulation has been sparse but consistent over the last two decades. Most research has come from the healthcare sector. Lehaney and Paul (1994a and 1994b) started the conversation about SSM's relevance in simulation projects. Lehaney and Huplic (1995) consider simulation's use in healthcare resource planning, and state that SSM could be feasible for guiding research agendas.

Lehaney et al (1999) developed an SSM structure which includes an account of the activities undertaken to develop a simulation project of an outpatient medical clinic. The authors refer to the approach as "soft simulation" and provide discussion about some of the challenges and advantages of using SSM in simulation.

Pidd (2007) presents many "soft" PSMs for "hard" simulation projects, and then focuses on root definitions to aid conceptual modeling. He draws upon an experience of simulation modeling carried out in a police department - one of the few cases of SSM in simulation not developed in the healthcare sector. The author argues that SSM enables a greater appreciation of a simulation project's context and the conceptual model's simplifications.

Checkland (1999) asserts that SSM is best applied in messy problems which are difficult to define by hard modeling techniques (such as linear or non-linear programming) and/or when multiple perspectives exist on system definition. Based on the fact that CM in simulation involves gathering various perspectives of the same investigation system and abstracting a workable model from an operational system, it would seem that SSM is a natural fit for CM in simulation projects. 


\section{Montevechi and Friend}

\section{A RUN-THROUGH OF CONVENTIONAL SOFT SYSTEMS METHODOLOGY TOOLS}

Instead of jumping directly to SSM applications in simulation projects, it is worth reflecting on the methodology's overall scope in order to facilitate later examples. Almost all of the information contained in this sub-section can be found in Checkland (1999) and Checkland and Scholes (1999).

Most SSM studies start by examining the transformation process, when the researchers identify what it really is that the investigation system does. Then, the components of the mnemonic CATWOE are identified. This helps define who benefits or suffers from the process's output (Customers); who carries out the transformation process (Actors); what transformation occurs from the system (Transformation); the context in which that transformation becomes something meaningful (Weltanschauung in German, or World View); the person who can stop the system (Owner); and those effects from outside the system which exercise a direct effect on system performance (Environmental Constraints), respectively. It should be noted here that this information is generally acquired through meetings and interviews with individuals within the system under study.

Based on this analysis, a root definition is then created, which can be compared to a business's mission statement (Kotiadis 2007). In other words, it is a short paragraph which connects the CATWOE components and verbalizes the investigation system's purpose and context. Checkland and Scholes (1999) point out that this root definition describes the ideal system and not the actual system.

With the ideal system defined, the next conventional SSM phase is to define the studied system's performance measures (efficacy, efficiency and effectiveness). By doing so, researchers are able to then measure or quantify the investigation system's performance and propose desirable and feasible alterations in order to bring the real system closer to the ideal system.

After these phases, what Checkland refers to as a SSM "conceptual model" - and what this paper refers to as a Purposeful Activity Model (PAM) to avoid confusion - can then be drawn up to describe the system in the minimum number of activities possible and visually represent the system dependencies and contingencies.

Upon a reassessment of the system performance measures, the entire cyclical process may start over again if the changes did not bring about the desired effects. It is in this point that Checkland and Scholes (1999) and Kotiadis (2007) assert that SSM facilitates the action-research agenda, as researchers and system specialists can analyze and make changes throughout the entire simulation project by revisiting points which could be improved within the model and the real system.

\section{5-STEP SOFT SYSTEMS METHODOLOGY FRAMEWORK TO GUIDE CONCEPTUAL MODELING}

The following sub-sections present relevant work in the area of simulation with SSM, while also proposing the fusion of two investigations (Kotiadis 2007, Lehaney and Paul 1996) which addressed different phases of Robinson's (2008b) CM framework.

\subsection{Developing System Understanding through SSM}

Based on the knowledge acquired and derived from system understanding, simulation practitioners construct conceptual models (Kotiadis and Robinson 2008). It can therefore be concluded that, in order to create a sound conceptual model, researchers must fully understand the system that they investigate. Doing so often involves viewing a system both as a whole, and with respect to its individual parts. For example, it would be convenient to view a production line as simply that; however, in many cases there are multiple "sub-systems" which participate in the system directly or indirectly and influence overall system performance (i.e. Quality Control, Internal Logistics).

Accurately modeling interdependencies is a key to a valid simulation project; PAM, a SSM analysis tool described above, aims to describe a system's main activities in the smallest number of activities possible (Checkland 1999). In order to build a PAM, SSM users must first define the system under study using SSM's systems analysis techniques. 


\section{Montevechi and Friend}

By first using conventional SSM techniques such as CATWOE analysis and root definitions, Lehaney and Paul (1996) designed a PAM to gain greater system understanding and differentiate between interrelated systems. In their work, SSM was used to select an investigation system to be modeled from a variety of healthcare systems (records, support and clinical); researchers "teased out" (Lehaney and Paul 1996) system details from clients using structured debate methods and sketching out PAMs. Based on the subsystem level of resolution, researchers were able to create a PAM, visualizing the activities which overlapped between the three systems, and thus aiding in the selection of relevant conceptual model components and the exclusion of activities which did not belong in the scope of the simulation project. The authors also remarked that SSM helped guide the data collection process.

In their study, Lehaney and Paul (1996) state that SSM helped consolidate the researchers' system understanding and knowledge acquisition. The output of this first model often will not have a sufficient level of resolution to guide the entire conceptual modeling process; it only provides an initial stepping stone from which researchers will be able to contextualize and orient their study. A more detailed model must be drawn up, which will be revisited in sub-section 4.2.

It should be noted here that Lehaney and Paul (1996) focused on system definition (phase I of Robinson 2008b); however, the authors pay little attention to developing project objectives (phase II of Robinson 2008b).

\subsection{Developing Simulation Project Objectives through SSM}

Following the CM phases proposed by Robinson (2008b), the next CM phase is to define the project's objectives. In order to do this, Kotiadis (2007) utilized SSM and developed an extension to PAM called a Performance Measurement Model (PMM) in an outpatient healthcare clinic. After elaborating the performance measures, the author proposed the creation of a PMM, which consists of five steps to be taken to generate simulation objectives:

(1) Find out how performance criteria relate to the real-life situation.

(2) Break performance criteria into specific monitoring activities which involve observing and recording information. The activities should be in the format 'Monitor...'

(3) Consider what action to take based on the monitoring activities. Record in the format 'Determine if...'

(4) Where possible, list monitoring activities first and 'determine if' activities second. Use letters and not numbers, to differentiate from the PAM.

(5) Consider which measurement activities can and should be evaluated by the simulation. The selected performance measurement activities form the objectives.

In her outpatient healthcare clinic study, she detailed the following performance measures:

- Efficacy: To check that the Intermediate Healthcare (IC) function is supported through IC strategic and operational activities,

- Efficiency: To check that the minimum IC resources are used to support the strategic and operational activities,

- Effectiveness: To check that the strategic and operational activities enable older people to be rehabilitated in the most appropriate service for their needs.

Following her proposal, the author then took these criteria and identified the activities which would need to be monitored in order to determine system performance (e.g. Monitor Capacity and Resources, Monitor Funding, Monitor Compliance with Healthcare Standards), and the contingent decisions that would have to be taken based on this monitoring (Determine if more resources will be necessary, Determine if processes need improving). Based on this verbalization, the author was then able to lead a more 


\section{Montevechi and Friend}

insightful discussion with the healthcare management into the questions which would be examined, aside from identifying which questions could indeed be investigated using simulation projects. In doing so, she filtered out irrelevant questions and channeled the collaborative efforts into the most feasible ones.

Further details on this approach may be found in Kotiadis (2007). It should be noted here that, aside from generating an initial PAM of the investigation system, the author puts forward a structured approach for defining simulation objectives (phases I and II of Robinson 2008b); however, she does not evaluate the creation of conceptual model content (phases III, IV and V of Robinson 2008b).

\subsection{Developing Model Inputs, Outputs and Content through SSM}

Once the simulation project's objectives are defined, the CM process moves into greater level of detail, dealing with its inputs, outputs and content (Phases III, IV and V of Robinson 2008b). Within the context of SSM in CM for simulation, phases III and IV of Robinson's (2008b) CM framework have not been directly addressed in the literature, according to the authors' evaluation of existing literature. However, in this article, it is proposed that, if simulation researchers follow the agenda proposed herein, the CATWOE analysis performed in previous steps will already have identified the system's input-output transformation process. Identifying the Actors and Customers will also help identify the individuals (and their corresponding resources, such as equipment and work stations) that participate in or influence the investigation system. Addressing the CATWOE analysis a second time in phases III and IV will enable researchers to identify the inputs (which many times are machines, work posts or reception areas, along with individuals who perform tasks at these locations). The objectives identified in phase II will then guide the context through which these inputs are evaluated. The model output will generally be the investigation system's transformation process output (i.e. patient needing care into treated patient); according to Robinson (2008b), the purpose of the model outputs is two-fold: it both evaluates if modeling objectives are being reached, and if they are not, they serve to help researchers figure out the reason for the discrepancy.

For the final CM phase, defining model content, Lehaney and Paul (1996) expanded certain strategic level activities from their PAM of the health clinic to include operational level activities. For example, in their study, they expanded the PAM activity "Provide planned resources for the Care of the Elderly Clinic" (a general, strategic level activity) to include the operational actions which are carried out by the clinical system Actors on the hospital floor: Provide Blood Test, Provide ECG, Provide X-Ray, and Provide Clinic. In doing so, the authors achieved a greater resolution in which to conduct meaningful conversation about the activities that would be included in the conceptual model, aside from identifying where they would have to collect data for the simulation model.

Another benefit of the PAM activity expansion technique is that, when there are multiple interconnected systems, the researchers will be able to identify which activities from each system need to be included in the model and which can be discarded. This will help define the scope of the model as well as the assumptions, as the researchers will be "assuming" that certain activities are not essential for successful project development.

\subsection{Developing a Communicative Model through SSM}

Communicative models help simulation practitioners transmit the understanding which has been acquired of the system by visual means (Balci 2003). This can be via a chart, diagram, image, etc. It is held by the authors of this article that, guided by Kotiadis's (2007) objective definition approach and based on the CATWOE analysis and PAM activity expansion from Lehaney and Paul (1996), researchers will easily be able to identify common communicative model components, such as: Processes and Transportation (activities which Actors perform); Resources (Equipment which Actors need to perform processes); and Entities (Inputs transformed into Outputs by Actors), just to name a few examples. These conceptual model components belong to the modeling technique IDEF-SIM from Montevechi et al. (2010). 


\section{Montevechi and Friend}

It should be mentioned here that, although no formalized approach has been found in the literature for transforming SSM analysis tools and models into conceptual model components, this process should be fairly intuitive, based on the identification of system components using Checkland's (1999) methodology.

\subsection{Conceptual Model Validation through SSM}

One essential CM phase which cannot be left out is validation. Sargent (2011) defines conceptual model validation as "determining that the theories and assumptions underlying the conceptual model are correct and that the model representation of the problem entity is 'reasonable' for the intended purpose of the model."

SSM may help in this point due to the fact that the CM process and its components are tracked with a structured, transparent logic, as researchers and systems specialists can sit down and ask questions, starting with the conceptual model itself, and referring back one model (or phase) at a time. In a sense, researchers and specialists are tracing back over the steps they have already taken. Imagine the conversation:

System Specialist: "Why is this routine quality inspection activity considered in the model? And why didn't we include maintenance routines? Weren't we going to evaluate just throughput and production per machine?"

Researcher: "If you'll recall, we included that because it belongs to the activities that compose the quality department support system inside the global production system, as we saw in the expanded PAM (phase V). And if you'll remember, the maintenance routines were excluded when we expanded the quality department's PAM, because we realized this only happens when the line isn't in operation on the weekends."

System Specialist: "And why are we examining quality inspection routines in the first place?"

Researcher: "Because the impact of alterations in throughput and the cost incurred due to quality inspections were decided to be one of the model's outputs (phase IV). The time spent doing each quality inspection and the intervals are our inputs which we are altering in the model to evaluate the impact (phase III). This is in line with the objectives we all agreed on for the PMM (phase II), which is in line with the global system definition in the PAM of the production system (phase I)."

System Specialist: "Oh, ok. I get it."

This simplistic, albeit real, example of an exchange in a face to face validation serves to show how SSM, and the adaptations proposed by Lehaney and Paul (1996) and Kotiadis (2007), help structure the logic and make the conceptual model's contents, and their reason for being included (or excluded), transparent for the clients. This, in turn, increases the model's credibility, which is essential to turning a simulated solution into a real one (Robinson 2008a).

\section{A STREAMLINED SSM APPROACH TO GUIDE THE CONCEPTUAL MODELING PROCESS}

Considering the review presented above, the following steps can be suggested for using SSM to guide the conceptual modeling process.

I. Using conventional SSM tools, develop a PAM of the system (or systems) to be studied along with Lehaney and Paul's (1996) approach for modeling strategic activities. This provides general system understanding (phase I), but not the operational resolution needed for conceptual model components.

II. Once performance measures have been identified, extend the strategic level activities PAM to include a PMM (Kotiadis, 2007). Following the steps presented generates the simulation study objectives (phase II). 


\section{Montevechi and Friend}

III. Use initial SSM tools to guide discussion on model output (phase III), input (phase IV), then expand the strategic activities from Phase I to include operational level activities, thus stimulating greater resolution and facilitating discussion about model scope and detail (phase V).

IV. Transform SSM models into a visual abstraction (communicative model) of the system using a conceptual modeling technique.

V. Conceptual Model Validation - (done in parallel with steps 1 to 4 above). If necessary, hold a meeting to retrace the steps which have been taken to develop the model.

\subsection{Synopsis of an Application of the proposed SSM approach to guide Simulation Conceptual Modeling}

Although this paper's aim is to contribute to CM theory through the proposal of a step-by-step SSM approach, it is worth mentioning that a statistically validated simulation model was in fact developed based on a validated conceptual model designed using the SSM approach herein described in a manufacturing environment. The examples given in this text originate from a real-world, action-research study of a production line belonging to an international company in the automotive components sector with a factory located in southeast Brazil. In following is a brief synopsis of the CM phase, which was guided the proposed SSM approach.

I. The company's management originally wanted to evaluate the impact of adding three new, automated machines to replace six manual operation assembly machines on overall line throughput. There was no doubt that the production process would be simulated; however, there was much doubt about which activities truly belonged to that system. Through CATWOE analysis and root definition verbalization, an initial PAM of the entire operational system was created in which three sub-systems were identified (phase I): Production, Quality and Inspection, and Internal Logistics. After initially dividing the investigation system's main strategic tasks into sub-systems, it was easier to see where their activities overlapped and converged. At this point, managers and researchers were able to see that the model would be able to tackle more profound questions about the system's dynamic behavior rather than simply analyzing throughput.

II. After developing traditional SSM performance measures of Efficacy, Efficiency and Effectiveness, a PMM was developed in order to visualize which questions could be addressed by the simulation project using Kotiadis' (2007) approach. For example, the activity "Monitor Costumer Complaints" and its contingent decision "Determine if special training is needed" were discarded from the simulation, as the managers believed the productive process quality gains would be difficult to quantify in the model. On the other hand, "Monitor Costs" and one of its contingent decisions, "Determine if Material Handler is necessary" was maintained in the model; the impact of the additional worker (Material Handler) on throughput became a central question of the study. This step led to the model's objectives (phase II).

III. The model output (phase III) was determined to be two-fold: Firstly, throughput and secondly, time spent executing quality control inspections. Then, model inputs (phase IV) were deemed to be the number of certain operators, equipment and the time interval between quality inspections. Then, by reviewing CATWOE analysis and root definitions developed earlier, the focus moved to model components (phase 5). Following Lehaney and Paul's (1996) example, the original strategic PAM was expanded to include operational level activities. This guided a rich discussion on which activities to include in or exclude from the model. As an example, the Quality and Inspection sub-system included the strategic activity "Carry Out Quality Control Routines". This box was expanded to include activities such as "Perform Routine Maintenance", "Perform Corrective Maintenance" and "Perform Speed Tests". This level of operational resolution enabled a more precise conversation about the model's pertinent activities. Continuing the given example, routine maintenance was excluded from the model because management commented that this only 


\section{Montevechi and Friend}

happened when the line was not operating on the weekends; corrective maintenance was deemed to be a machine break-down rule to be included per machine (thus identifying a data collection point); and hourly quality checks would be included as an activity to be performed at designated quality-control work benches, thus becoming an important part of the model.

IV. Once all of these model components were identified, a conceptual model was constructed using the modeling technique IDEF-SIM proposed by Montevechi et al (2010). Seeing as model components such as resources (Actors) and activities (Transformation) had already been identified, SSM definitions and models were readily transformed into the conceptual model.

V. The validation process occurred naturally throughout each step of the process, according to the managers. As they had been shown each SSM developed by the researchers, they were confident of their understanding of the system. Upon some insistence by the researchers, a final face-toface validation meeting was held, where the model was presented and validated (on a side note, the conversation in sub-section 4.5 really took place).

Aside from the confidence the managers had in the model due to SSM's transparency, it was possible to note the sense of participation the managers held in the researchers' knowledge and understanding of the situation. The inclusion was especially powerful with the internal logistics and quality department managers, who both made the same comment: “This isn't just production's model. This is our model - the company's model."

Fourteen different work posts and ten employees per shift (three shifts) were modeled using simulation software. Aspects involving the pull-style production system, work in progress, machine breakdowns, and employee breaks were programmed using a commercial software package. Real production data were compared with simulated data, and a Two-Sample T test was applied to compare the values; the model proved to be statistically robust in simulating line throughput. Among other questions about staffing decisions and internal logistics, the company's current focus is to use the model to evaluate the impact of quality inspection routine interval scenarios on throughput and operational costs.

Finally, this action-research simulation research project was developed in conjunction with the master's dissertation Friend (2012), which proposed the use of knowledge acquisition techniques (SSM) and knowledge management strategies, such as data organization in formats which are congruent with simulation software requirements, in order to facilitate CM, implementation and simulation projects in general. Research in this manufacturing environment with this particular model and project continues to be developed both academically and in practice with the help of the company's management team. Due to confidentiality policies, the authors were asked to conceal the name of the company and its product.

\section{DISCUSSION}

For the purposes of the mentioned action-research project, it seems SSM served as a good compass to establish the direction and rhythm of the project. From the outset, the researchers had a well-defined agenda to follow and knew which questions they wanted to ask and how to find their responses. Nonetheless, this is not to say that SSM is a panacea for all CM processes, and further research should be conducted using the methodology in CM and simulation projects in the numerous sectors in which simulation may be applied . Kotiadis (2007) had noted that all SSM applications in simulation projects had previously come from the healthcare sector.

Conceptual modeling is the first of three stages of simulation projects, as defined by Montevechi et al. (2010). Once the conceptual model is validated, the project proceeds to the subsequent stage, Implementation, which involves the translation of the system abstraction (conceptual model) into a computational model, generally programmed using a simulation software package.

In the implementation phase, a computerized representation of the conceptual model is fed the data collected for the study. Upon validation of the computerized model, experiments can be run using optimization software in the analysis phase. Although this paper focused on SSM in CM, these conventional 


\section{Montevechi and Friend}

simulation project phases can also be facilitated by SSM as well; for example, by referring to the original questions formed using Kotiadis's (2007) method to arrive at project objectives, researchers will not lose sight of their goal as experimental factors are chosen for optimization.

Perhaps the greatest contribution SSM makes to simulation projects is in its ability to structure the highly qualitative investigation process that takes place in the CM phase. The methodology provides a means by which to visually abstract knowledge acquired from systems specialists. As previously stated, the techniques used in this paper's SSM approach are not brand-new academic contributions; however, the molding of the two approaches into a single, step-by-step framework do constitute a new development. As seen in the action-research study synopsis, the framework was able to guide beginning and experienced practitioners in making the $\mathrm{CM}$ phase and project management more transparent, objective and efficient and, in turn, arriving at validated conceptual and computational models.

One important point in the simulation process after generating a conceptual model is data collection. SSM is not meant to tackle the question of data treatment itself, but it does identify where data can be found and collected through the identification of Inputs, Actors and their necessary equipment or work posts. For example, if machine operator $Z$ is identified as an Actor in a production system which processes the system's main entity, and it is known that he or she needs machine $X$ to complete the transformation process, then it should be recognizable that the processing time at machine $X$ needs to be analyzed for time data analysis. Thus, the initial CATWOE analysis and the subsequent operational activity expansion proposed by Lehaney and Paul (1996) lead researchers to identify who carries out the transformation process, where it is done and what equipment or work-posts are used. This identification should help reduce doubts about where to collect what type of data.

Following up on data collection, one shortcoming of the use of SSM in simulation is that its heavy focused on human interaction (which is a strong point for the methodology) does not directly address the question of physical goods or equipment necessary to complete a task (but instead the task or activity that an individual carries out through the use of such equipment). Generally, this equipment would constitute a location or resource within a conceptual model.

On a more qualitative note, SSM also serves to make the CM process more transparent. It gets people involved by asking who the Actors and Clients are for each system, aside from the context which makes the process meaningful (Weltanschauung). Pidd (2007) commented that SSM gave the department's participants a sense of ownership of the model in his police officer deployment simulation project. The model wasn't just for HQ or admin. It was their (the officers') model. This, theoretically, should promote greater participation and good will on behalf of those who belong to the investigation system.

\section{CONCLUSIONS AND RECOMMENDATIONS}

Conceptual modeling for simulation projects is an essential step which involves much discussion between simulation practitioners and systems specialists along with analysis of human and systemic interactions. As has been seen in the literature and this article, SSM serves as a guiding mechanism which captures and maps both where simulation projects are and where they want to go.

One recommendation for future work with SSM in CM would be the development of an extension which could capture the physical resources (i.e. a machine, a vehicle, a piece of medical equipment) that the Actors use to perform his or her activities (i.e. production, transportation, medical exam) and on which the system's transformation and output are contingent (parts produced, products transported, patients examined). This would enable simulation practitioners to capture an important detail that is currently only implicit when using SSM in CM. As mentioned in the previous section, this would also help with data collection by identifying not only the activities to be analyzed, but also the physical locations and equipment where time-analysis data would need to be collected.

A final recommendation for potential theoretical opportunities and practical applications would be the formalization of a procedure by which to transform SSM models and analyses into communicative conceptual models. That is, for now the connection between SSM models and CM techniques is merely im- 


\section{Montevechi and Friend}

plicit and not structured; an interface or logic would enable correlation between SSM Actors, Inputs and Transformation Processes, thus directly channeling SSM models into specific visual representations in a CM technique.

\section{ACKNOWLEDGEMENTS}

The authors would like to express their gratitude to PADTEC, CNPq, CAPES pro-engineering program, the Minas Gerais research foundation FAPEMIG for their continued support in this project.

\section{REFERENCES}

Abdulmalek, F.A., Rajgopal, J. 2007. "Analyzing the benefits of lean manufacturing and value stream mapping via simulation. A process sector case study." International Journal of Production Economics 107:223-236.

Balci, O. 2003. "Verification, Validation and Certification of Modeling and Simulation Applications." In Proceedings of the 2003 Winter Simulation Conference. Edited by S. Chick, P. J. Sánchez, D. Ferrin, and D. J. Morrice, 150-158. New Orleans, Louisiana: Institute of Electrical and Electronic Engineers, Inc.

Balci, O., Arthur J. D., and Nance R. E. 2008. "Accomplishing reuse with a simulation conceptual model." In Proceedings of the 2008 Winter Simulation Conference. Edited by S.J. Mason, R.R. Hill, L. Mönch, O. Rose, T. Jefferson and J.W. Fowler, 959-965. Miami, Florida: Institute of Electrical and Electronic Engineers, Inc.

Checkland, P. 1999. Systems Thinking, Systems Practice: Includes a 30-Year Retrospective. Chichester: Wiley.

Checkland, P., and Scholes, J. 1999. Soft Systems Methodology: A 30-Year Retrospective. Chichester: Wiley.

Chwif, L. and Medina, A.C. 2007. Modelagem e simulação de eventos discretos: teoria e aplicações. 3rd ed. São Paulo: Ed. dos autores.

Friend, J. D. 2012. "Aplicação de uma abordagem de aquisição e armazenamento do conhecimento em projetos de simulação a eventos discretos." Master's Dissertation, Department of Industrial Engineering, Federal University of Itajubá, Itajubá, Minas Gerais.

Kotiadis, K. 2007. "Using soft systems methodology to determine the simulation study objectives." Journal of Simulation 1:215-222.

Kotiadis, K. and Robinson, S. 2008. "Conceptual modelling: Knowledge acquisition and model abstraction." In Proceedings of the 2008 Winter Simulation Conference. Edited by S.J. Mason, R.R. Hill, L. Mönch, O. Rose, T. Jefferson and J.W. Fowler, 951-957. Miami, Florida: Institute of Electrical and Electronic Engineers, Inc.

Law, A.M. 1991. "Simulation model's level of detail determines effectiveness." Industrial Engineering 23:16-18.

Law, A.M. 2009. "How to build valid and credible simulation models." In Proceedings of the 2009 Winter Simulation Conference. Edited by M.D. Rossetti, R.R. Hill, B. Johansson, A. Dunkin, R.G. Ingalls, 24-33. Austin, Texas: Institute of Electrical and Electronic Engineers, Inc.

Lehaney, B., Clarke, S.A., and Paul R.J. 1999. "A case of an intervention in an outpatients department." Journal of the Operational Research Society 50:877-891.

Lehaney, B. and Hlupic, V. 1995. "Simulation modelling for resource allocation and planning in the health sector." Journal of the Royal Society of Health 115:382-385.

Lehaney, B. and Paul, R.J. 1994a. "Developing sufficient conditions for an activity cycle diagram from the necessary conditions in a conceptual model." Systemist 16:261-268.

Lehaney, B. and Paul, R.J. 1994b. "Using soft systems methodology to develop a simulation of outpatient services." Journal of the Royal Society of Health 114:248-251. 


\section{Montevechi and Friend}

Lehaney, B. and Paul, R.J. 1996. "The use of soft systems methodology in the development of a simulation of out-patients services at Watford general hospital." Journal of the Operational Research Society 47:864-870.

Karagöz, N.A. 2008. "A framework for developing conceptual models of the mission space for simulation systems." Ph.D. thesis, Center of Modeling and Simulation, Middle East Technical University, Ankara, Turkey. http://etd.lib.metu.edu.tr/upload/12609580/index.pdf [Accessed March, 7, 2012].

Montevechi, J.A.B., Leal F., Pinho A.F., Costa R.F.S. and Oliveira M.L.M. 2010. "Conceptual modeling in simulation projects by mean adapted IDEF: an application in a Brazilian tech company." In Proceedings of the 2010 Winter Simulation Conference. Edited by B. Johansson, S. Jain, J. MontoyaTorres, J. Hugan and E. Yücesan, 1624-1635. Baltimore, Maryland: Institute of Electrical and Electronic Engineers, Inc.

Pidd, M. 2007. "Making sure you tackle the right problem: Linking hard and soft methods in simulation practice." In Proceedings of the 2007 Winter Simulation Conference. Edited by S.G. Henderson, B. Biller, M.H. Hsieh, J. Shortle, J.D. Tew and R.R. Barton, 195-204. Washington, D.C: Institute of Electrical and Electronic Engineers, Inc.

Robinson, S. 2008a. "Conceptual modelling for simulation Part I: definition and requirements." Journal of the Operational Research Society 59:278-290.

Robinson, S. 2008b. "Conceptual modelling for simulation Part II: a framework for conceptual modelling." Journal of the Operational Research Society 59:291-304.

Sargent, R. 2011. "Verification and Validation of Simulation Models." In Proceedings of the 2011 Winter Simulation Conference. Edited by S. Jain, J. Creasey, K.P. Himmelspach, White and M. Fu, 166-183. Phoenix, AZ.

Wang, W. and Brooks, R.J. 2007. "Improving the understanding of conceptual modelling." Journal of Simulation 1:153-158.

\section{AUTHOR BIOGRAPHIES}

JOSÉ ARNALDO BARRA MONTEVECHI is a Titular Professor of the Industrial Engineering and Management Institute (Instituto de Engenharia de Produção e Gestão) at the Federal University of Itajubá in Brazil. He obtained his undergraduate, master's and doctorate degrees, respectively, in Mechanical Engineering from the Federal University of Itajubá, the Federal University of Santa Catarina, and the Polytechnic School at the University of São Paulo. His research interests include Operational Research, Simulation and Economic Engineering. His e-mail address is montevechi@unifei.edu.br.

JONATHAN DANIEL FRIEND is a Researcher in the Industrial Engineering and Management Institute (Instituto de Engenharia de Produção e Gestão) at the Federal University of Itajubá in Brazil. He obtained his bachelor's degree in Communication and Spanish at the University of Indianapolis and his master's degree in Industrial Engineering at the Federal University of Itajubá. His research interests include Operational Research, Simulation, Conceptual Modeling, and Logistics. His e-mail address is daniel.friend1@gmail.com. 